\title{
Variations in intensity of end-of-life cancer therapy by cancer type at a Canadian tertiary cancer centre between 2003 and 2010
}

\author{
Petra Grendarova • Aynharan Sinnarajah • \\ Theresa Trotter • Cynthia Card • Jackson S.Y. Wu
}

Received: 28 October 2014 / Accepted: 22 February 2015 / Published online: 7 March 2015

(C) The Author(s) 2015. This article is published with open access at Springerlink.com

\begin{abstract}
Background Aggressive medical management of cancer patients at the end of life (EOL) is an indicator of health services quality. We evaluated the variations in EOL cancer therapy utilization and in acute care hospital deaths across different types of cancer within the setting of a regionalized cancer program. Methods Intravenous chemotherapy and radiotherapy use within the last 14 and 30 days of life was identified through the Alberta Cancer Registry and then verified by chart review for cancer decedents residing within $50 \mathrm{~km}$ of the Tom Baker Cancer Centre between 2003 and 2010. Multivariable logistic regression was used to examine variations in outcomes of interest by cancer, adjusting for age and other factors in prespecified models.
\end{abstract}

This paper was presented in part at the Canadian Association of Radiation Oncology, September 2013, Montreal, QC.

Electronic supplementary material The online version of this article (doi:10.1007/s00520-015-2676-y) contains supplementary material, which is available to authorized users.

P. Grendarova $\cdot$ T. Trotter $\cdot$ J. S. Wu $(\bowtie)$

Department of Oncology, Division of Radiation Oncology, Tom Baker Cancer Centre, University of Calgary, Calgary, AB, Canada e-mail: jackson.wu@albertahealthservices.ca

P. Grendarova

Clinician Investigator Program, Department of Community Health

Sciences, University of Calgary, Calgary, NW, Canada

A. Sinnarajah

Palliative and End of Life Care, Alberta Health Services,

Calgary, AB, Canada

\author{
A. Sinnarajah \\ Department of Oncology, Division of Palliative Medicine, Tom \\ Baker Cancer Centre, University of Calgary, Calgary, AB, Canada \\ C. Card \\ Department of Oncology, Division of Medical Oncology, Tom Baker \\ Cancer Centre, University of Calgary, Calgary, AB, Canada
}

Results Of the 9863 decedents included in the study, 3.0 and $6.3 \%$ received chemotherapy within the final 14 and 30 days of life, respectively. In multivariable model, breast, hematological, and gynecological cancers were at least 2.5 times more likely than other cancers to undergo EOL chemotherapy. Radiotherapy was given to $4.6 \%$ of decedents within 14 days of death, but only $66 \%$ (359/542 courses) were completed as prescribed. Acute care admission within 14 days of death was seen in $44 \%$ of decedents and $34 \%$ died in the hospital. Conclusions In our regional cancer program, the intensity of cancer therapies near the end of life varied considerably across different cancer types. Such variations may be unwarranted. A substantial proportion of cancer deaths occurred in the acute care setting. Greater efforts to integrate palliative care in outpatient cancer services are needed.

Keywords End-of-life care $\cdot$ Palliative oncology $\cdot$ Health services research $\cdot$ Quality care

\section{Background}

In "The Quality of Death: Ranking End-of-life Care across the World" report published in 2010, the Economist Intelligence Unit remarked that 'the 'medicalisation' of death in Canada has engendered a culture where many people are afraid to raise the topic of death" [1]. This "medicalisation" can be seen in the growing intensity and aggressiveness of care involving cancer therapies and acute care services for cancer patients near the end of life. Cancer therapies, such as chemotherapy and radiotherapy, are typically delivered within prescribed protocols, with adverse effects deemed tolerable among patients with adequate functional performance or physiologic reserve for physical recovery. But for those with end-stage 
disease, when management goals are geared towards symptom control and comfort, the burden of treatment logistics and side effects can be profound and can adversely impact the quality of life.

Indicators of aggressive end-of-life cancer care and their benchmarking for health system performance were first proposed by Earle et al. [2, 3]. Overly aggressive care is evident when measures such as chemotherapy given within last 14 days of life, new chemotherapy initiated within last 30 days of life, and acute care hospital death exceed benchmarks. Those metrics have been expanded to include end-of-life radiotherapy and they allow examination of variations in their utilization across regions and practice types in the USA [4-7]. Observations have also been reported for cancer decedents in Ontario between 1993 and 2004, where variations in the aggressiveness of therapies and services were observed across five cancer types, with a trend towards increasing end-of-life chemotherapy over time [8].

Oncology practice in Canada often involves multidisciplinary care teams (as "tumor groups" or "disease site groups"), and clinical practice guidelines are usually concerned with treatment indications for the average well patient, but relatively limited in scope for the highly heterogeneous palliative cancer population. Decisions to initiate or continue cancer therapies in this setting may be driven by perceived rather than established needs and benefits, which could lead to unwarranted variations in intensity and aggressiveness of care. Thus, we were first interested in examining the use of end-of-life cancer therapy and location of death for cancer patients managed through our regional program, and second, we evaluated variations in those outcomes across a broad range of cancer types, controlling for some relevant factors. Confirmation of variations in the intensity of end-of-life care among different cancer types would be informative to multidisciplinary practices and facilitate attention on practice culture.

\section{Materials and methods}

Health system and study setting

Alberta has a multicultural population with $20 \%$ visible minority and aboriginal population (Canada 2006 Census). Two provincial comprehensive cancer centers provide services for adult malignancies to a population of 3.5 million residents, one of them in Calgary (Tom Baker Cancer Centre, TBCC). TBCC processes about 5000 incident cancers per year, the majority via outpatient visits for assessment and medical treatment coordinated within multidisciplinary groups comprised of medical specialists and other health professionals, for which practice guidelines are developed and maintained [9]. However, palliative care specialists are not an integral part of each multidisciplinary team in the outpatient setting; rather, specialized palliative clinics (mainly for pain and symptom management) run separately from subspecialized tumorspecific clinics. This study was conducted solely within the TBCC practice environment given data availability and that standards were not yet in place to allow for province-wide comparison. This study was approved by institutional review board (Alberta Cancer Research Ethics Committee no. 25968).

Inclusion and exclusion

This is a retrospective health services study of decedents deemed to have cancer as the main cause of death between Jan 2003 and Dec 2010. Cases over 18 years of age at time of cancer diagnosis were included, regardless of cancer stage at diagnosis, if the residence of death or last known postal code were within $50 \mathrm{~km}$ driving distance from TBCC, and their deaths were located in the Calgary region, in order to limit variations in utilization due to geographic distance [10]. For the planned analyses, cases without cancer clinic registration and rapidly fatal disease (cancer diagnosed within 1 month of death) were excluded. Supplementary Fig. 1 summarizes the selection.

\section{Data sources and verification}

The Alberta Cancer Registry (ACR) is a provincial cancer registry with gold certification yearly by the North American Association of Central Cancer Registries [11], maintained by the Cancer Management Outcomes Research and Evaluation, which also collects administrative data for service delivery through provincial cancer care electronic medical records. ICD-10 cause-of-death (vital statistics report) is recoded where additional information is available to the ACR coder and matched to registry ICDO-3 diagnosis (99 \% match to cancer site fine level). The ACR performed retrieval of all recorded cancer decedents for this study in October 2012, along with their radiotherapy and medical day care appointments within 30 days of death. For those cases with medical day care treatments (i.e., systemic therapy), delivery of intravenous chemotherapy agents was further verified through TBCC pharmacy dispensary records. (Only intravenous therapies, including classical cytotoxic agents as well as monoclonal antibodies and proteasome inhibitors, were included because of their expected toxicities and/or demand on health system resources for administration.) Radiotherapy treatment delivery were verified through treatment planning and electronic records for treatment volume, fractions prescribed, and course completion. Chemotherapy delivered through any of the three acute care facilities in Calgary was queried through data linkage with hospital patient clinical information databases (SCM) and regional data management department (DIMR), using provincial unique health identifier. This linkage also provides data on acute care admissions before death. 
Variables and outcomes of interest

The primary outcomes of interest in the study include end-oflife (defined as within 14 days of death) chemotherapy and radiotherapy utilizations (two separate outcomes) and death occurring in acute care facility (hospital death). Secondary outcomes include new chemotherapy within 30 days of death, any chemotherapy within 30 days of death, and radiotherapy within 30 days of death. Independent variables (covariates) included cancer type, age at death, year of death, sex, disease duration, and comorbidity as known predictors [8, 12]; in addition, acute care admission within 14 days of death was included as a covariate (yes/no) to approximate clinical acuity. Registry cancers (73 sites) were recategorized into common cancer types and their respective multidisciplinary tumor groups. Disease duration, potentially a marker of aggressive or advanced disease, was measured as time between date of cancer diagnosis and date of death. Comorbidity was represented using the Deyo adaptation of the Charlson comorbidity score, which looks at the last year of all inpatient/emergency admissions and diagnosis data [13, 14]. Registry coding of collaborative cancer stage (as opposed to clinical cancer stage) was still undergoing implementation during the study period and not sufficiently complete for inclusion as a covariate.

\section{Statistical analysis}

Outcomes and covariates were summarized by tables and frequency distributions for categorical and continuous variables as appropriate. Variations in primary outcomes by cancer type were examined in prespecified multivariable logistic regression models. For end-of-life chemotherapy and end-of-life radiotherapy outcomes, the seven covariates of interest were cancer type, age, disease duration, sex, comorbidity, acute care admission, and year of death. For modeling of hospital death outcome, the same covariates were used, except that acute care admission before death was replaced by exposure to end-of-life therapy (chemotherapy and/or radiotherapy) to examine for association. Apart from the hypothesized cancer type effect on outcomes of interest independent of prespecified covariates, no additional hypothesis was tested; statistical inferences are presented and interpreted based on multivariable model point estimates (odds ratios, OR) and $95 \%$ confidence limits (CL). Computed results are rounded to two scientific digits in the text for simplicity. Because of low event rates and numerous covariate levels, Firth's penalized likelihood method was used to correct for small sample bias in the end-of-life chemotherapy and radiotherapy models. The absence of interaction between cancer type and age was confirmed within respective models; no other interaction was prespecified for testing. Model goodness-of-fit was checked using Hosmer-Lemeshow partitioning. Statistical analyses were performed using SAS 9.2 (SAS Inc., Cary, NC).

\section{Results}

\section{Decedents' characteristics}

Between 2003 and 2010, 9863 decedents met the study inclusion criteria, with a mean age of 70 years at time of death and a slight preponderance towards male deaths. The top five cancer causes of death by tumor groups were gastrointestinal $(27 \%)$, lung $(24 \%)$, genitourinary (11\%), hematologic $(9.1 \%)$, and breast cancer $(8.8 \%)$. Twenty-nine percent (2907/9863) of all decedents were diagnosed between 1 and 6 months before death. The characteristics of decedents and their respective outcomes are summarized in Table 1 (outcomes by 18 cancer types are provided in Supplementary Table 1).

\section{End-of-life cancer therapy and location of death}

Of the medical day care treatment encounters identified through administrative records, only $64 \%$ were verified to have received chemotherapy. Including acute care in-patient records of chemotherapy use $(n=65)$, the overall end-of-life chemotherapy utilization was $3.0 \%$ (296/9863). The use of a new chemotherapy agent within 30 days of death was $2.2 \%$ (220/9863), while any chemotherapy with 30 days of death was $6.3 \%(622 / 9863)$.

End-of-life radiotherapy use was accurately captured through administrative data in $95 \%$ of cases, giving a rate of $4.6 \%$ (458/ 9863 ); radiotherapy use within 30 days of death was seen in $10 \%(988 / 9863)$. The most common indication for radiotherapy was metastatic bone disease, followed by lung/mediastinal disease and brain metastases. Seventy-five percent of end-of-life radiotherapy prescriptions involved multiple fractions of treatments (almost always five or more fractions), but only $66 \%$ of all prescribed treatment volumes were completed as planned. The characteristics of radiotherapy are summarized in Table 2 . Trends over the 8 -year study period and by age for different cancer types for end-of-life chemotherapy and end-of-life radiotherapy are shown in Supplementary Figs. 2-5.

Forty-four percent (4362/9863) of decedents required acute care admission within 14 days of death. With respect to the location of death, the majority occurred in hospice facilities (41\%, 4074/9863) and in acute care hospital setting (34\%, $3355 / 9863)$. Nineteen percent (1902/9863) of the decedents had died at home and the remainder in long-term care (Table 1).

Variations in end-of-life cancer therapy and hospital death across cancer types

The variations in end-of-life chemotherapy and radiotherapy use across cancer types, controlling for age, disease duration, and other covariates, are shown in Figs. 1 and 2. Compared to other cancers, breast cancer (OR 3.3, CL 1.8-6.1), lymphomamyeloma (OR 3.3, CL 1.9-6.1), and gynecological cancers 
Table 1 Characteristics of study population and associated outcomes-of-interest

\begin{tabular}{|c|c|c|c|c|c|c|c|c|}
\hline & $\begin{array}{l}\text { Number of } \\
\text { decedents }(n)\end{array}$ & $\begin{array}{l}\% \text { of cohort } \\
\text { (column } \%)\end{array}$ & $\begin{array}{l}\text { EOL } \\
\text { chemo } \\
\text { (row\%) }\end{array}$ & $\begin{array}{l}\text { EOL RT } \\
\text { (row\%) }\end{array}$ & $\begin{array}{l}\text { New chemo } \\
\text { within } 30 \text { days } \\
\text { (row\%) }\end{array}$ & $\begin{array}{l}\text { Any chemo } \\
\text { within } 30 \text { days } \\
\text { (row\%) }\end{array}$ & $\begin{array}{l}\text { RT within } \\
30 \text { days } \\
\text { (row\%) }\end{array}$ & $\begin{array}{l}\text { Death in } \\
\text { hospital } \\
\text { (row\%) }\end{array}$ \\
\hline Cohort & 9863 & 100 & 3.00 & 4.64 & 2.23 & 6.3 & 10.0 & 34.0 \\
\hline Age at death & \multicolumn{8}{|c|}{ Median 71, mean 70, IQR 19, range 19-103 } \\
\hline $18-44$ & 417 & 4.2 & 7.4 & 7.0 & 3.6 & 14.2 & 13.0 & 44.6 \\
\hline $45-54$ & 1098 & 11.1 & 5.4 & 6.7 & 4.0 & 10.5 & 13.6 & 38.3 \\
\hline $55-64$ & 1906 & 19.3 & 4.0 & 5.6 & 3.1 & 8.3 & 12.2 & 37.4 \\
\hline $65-74$ & 2508 & 25.4 & 3.6 & 4.7 & 2.8 & 7.8 & 10.8 & 36.0 \\
\hline $75-84$ & 2777 & 28.2 & 1.4 & 3.6 & 1.1 & 3.0 & 8.1 & 29.9 \\
\hline $85+$ & 1157 & 11.7 & 0.3 & 2.7 & 0.2 & 0.8 & 4.9 & 26.1 \\
\hline \multicolumn{9}{|l|}{ Sex } \\
\hline$\%$ female, \%male & 9863 & $48.7,51.3$ & $3.0,3.0$ & $4.1,5.2$ & $2.4,2.1$ & $6.4,6.2$ & $9.4,10.6$ & $32.1,35.9$ \\
\hline \multicolumn{9}{|l|}{ Cancer type } \\
\hline GI & 2682 & 27.2 & 2.8 & 1.6 & 1.8 & 6.9 & 4.0 & 29.9 \\
\hline Colorectal $^{\mathrm{a}}$ & 1134 & 11.5 & 3.3 & 1.2 & 2.0 & 6.5 & 4.1 & 30.3 \\
\hline HepatoPanc $^{\mathrm{a}}$ & 927 & 9.4 & 2.9 & 0.7 & 1.9 & 9.6 & 1.5 & 28.4 \\
\hline GI others ${ }^{\mathrm{a}}$ & 621 & 6.3 & 1.9 & 3.7 & 1.0 & 3.5 & 7.6 & 31.2 \\
\hline Lung & 2364 & 24.0 & 2.2 & 8.3 & 2.2 & 4.3 & 17.6 & 34.3 \\
\hline GU & 1099 & 11.1 & 1.6 & 3.9 & 1.1 & 3.6 & 8.6 & 34.8 \\
\hline Prostate & 584 & 5.9 & 1.8 & 4.5 & 1.4 & 4.1 & 10.3 & 37.7 \\
\hline GU others & 515 & 5.2 & 1.5 & 3.4 & 0.9 & 3.1 & 7.2 & 32.2 \\
\hline Hematologic & 899 & 9.1 & 5.8 & 4.3 & 2.7 & 11.7 & 8.1 & 52.4 \\
\hline Leukemia & 293 & 3.0 & 4.1 & 1.4 & 1.7 & 7.2 & 3.1 & 55.6 \\
\hline Lymphom-myelom & 606 & 6.1 & 6.6 & 5.8 & 3.1 & 13.9 & 10.6 & 50.8 \\
\hline Breast & 864 & 8.8 & 5.3 & 3.9 & 3.5 & 9.3 & 10.2 & 32.3 \\
\hline Gyne & 542 & 5.5 & 4.6 & 2.6 & 4.6 & 9.2 & 5.4 & 34.1 \\
\hline CNS & 355 & 3.6 & 0.0 & 4.2 & 0.0 & 0.3 & 7.3 & 19.2 \\
\hline Head and neck & 306 & 3.1 & 3.3 & 5.9 & 1.6 & 5.9 & 14.7 & 38.9 \\
\hline Others & 752 & 7.6 & 2.4 & 7.3 & 3.3 & 5.7 & 14.4 & 31.9 \\
\hline Disease duration & \multicolumn{8}{|c|}{ Median 1.1 years, mean 2.6, IQR 2.5, range $0.1-50.3$} \\
\hline $1-3$ months & 1553 & 15.8 & 4.0 & 8.8 & 4.1 & 7.9 & 17.5 & 40.8 \\
\hline $3-6$ months & 1354 & 13.7 & 3.4 & 5.5 & 2.3 & 7.8 & 10.8 & 34.6 \\
\hline 6-12 months & 1792 & 18.2 & 2.7 & 4.2 & 1.6 & 6.0 & 9.3 & 32.6 \\
\hline $1-2$ years & 1819 & 18.4 & 2.6 & 3.3 & 2.0 & 5.7 & 7.9 & 30.8 \\
\hline $2-5$ years & 1831 & 18.6 & 3.1 & 3.6 & 2.0 & 6.2 & 8.6 & 32.3 \\
\hline$\geq 5$ years & 1514 & 15.4 & 2.4 & 3.0 & 1.5 & 4.6 & 6.9 & 34.2 \\
\hline \multicolumn{9}{|l|}{ Year of death } \\
\hline 2003 & 1080 & 11.0 & 4.4 & 6.0 & 3.2 & 8.2 & 11.0 & 38.2 \\
\hline 2004 & 1162 & 11.8 & 2.5 & 5.1 & 2.1 & 6.2 & 10.4 & 38.3 \\
\hline 2005 & 1163 & 11.8 & 3.0 & 5.6 & 2.4 & 5.6 & 11.9 & 33.1 \\
\hline 2006 & 1276 & 12.9 & 2.6 & 4.1 & 2.0 & 6.0 & 9.6 & 34.5 \\
\hline 2007 & 1269 & 12.9 & 2.5 & 5.3 & 1.5 & 6.2 & 10.6 & 32.9 \\
\hline 2008 & 1295 & 13.1 & 3.5 & 4.8 & 2.1 & 6.4 & 10.7 & 32.1 \\
\hline 2009 & 1320 & 13.4 & 2.6 & 3.7 & 2.1 & 5.7 & 9.5 & 31.7 \\
\hline 2010 & 1298 & 13.2 & 3.1 & 3.0 & 2.6 & 6.5 & 6.9 & 32.4 \\
\hline \multicolumn{9}{|c|}{ Hospitalization within 14 days of death } \\
\hline Yes & 4362 & 44.2 & 4.8 & 8.6 & 3.4 & 10.1 & 16.3 & $70.8^{\mathrm{b}}$ \\
\hline No & 5501 & 55.8 & 1.6 & 1.5 & 1.3 & 3.3 & 5.0 & 4.8 \\
\hline
\end{tabular}


Table 1 (continued)

\begin{tabular}{|c|c|c|c|c|c|c|c|c|}
\hline & $\begin{array}{l}\text { Number of } \\
\text { decedents }(n)\end{array}$ & $\begin{array}{l}\% \text { of cohort } \\
\text { (column } \% \text { ) }\end{array}$ & $\begin{array}{l}\text { EOL } \\
\text { chemo } \\
(\text { row } \%)\end{array}$ & $\begin{array}{l}\text { EOL RT } \\
\text { (row\%) }\end{array}$ & $\begin{array}{l}\text { New chemo } \\
\text { within } 30 \text { days } \\
\text { (row\%) }\end{array}$ & $\begin{array}{l}\text { Any chemo } \\
\text { within } 30 \text { days } \\
\text { (row\%) }\end{array}$ & $\begin{array}{l}\text { RT within } \\
30 \text { days } \\
\text { (row\%) }\end{array}$ & $\begin{array}{l}\text { Death in } \\
\text { hospital } \\
(\text { row } \%)\end{array}$ \\
\hline \multicolumn{9}{|c|}{ Comorbidity (Charlson score, Deyo adaptation; excludes cancer diagnosis) ${ }^{\mathrm{c}}$} \\
\hline 0 & 5987 & 60.7 & 3.1 & 4.7 & 2.4 & 6.5 & 10.3 & 29.1 \\
\hline 1 & 2060 & 20.9 & 2.7 & 4.9 & 2.1 & 6.2 & 10.6 & 37.5 \\
\hline$\geq 2$ & 1814 & 18.4 & 3.0 & 4.2 & 1.9 & 5.9 & 8.4 & 46.2 \\
\hline \multicolumn{9}{|l|}{ Place of death } \\
\hline Hospital & 3355 & 34.0 & 6.8 & 9.3 & 4.5 & 12.3 & 15.9 & - \\
\hline Hospice & 4074 & 41.3 & 0.6 & 1.9 & 0.7 & 2.6 & 7.0 & - \\
\hline Home & 1902 & 19.3 & 2.2 & 3.1 & 2.0 & 5.2 & 8.0 & - \\
\hline Long-term care & 532 & 5.4 & 0.6 & 1.7 & 0.2 & 0.8 & 3.0 & - \\
\hline
\end{tabular}

${ }^{\text {a }}$ Colorectal includes anal carcinomas; HepatoPanc includes the liver, biliary tract, and pancreas; GI others include intestinal and upper GI cancers

${ }^{\mathrm{b}} 29.2 \%$ of 4362 admitted patients were discharged from hospital before death: hospice $22.4 \%$, home $5.3 \%$, long-term care $1.5 \%$

${ }^{\mathrm{c}}$ Missing comorbidity scores in two cases

(OR 2.5, CL 1.3-4.8) demonstrate strong tendencies towards the use of end-of-life chemotherapy; colorectal cancer shows a relatively modest effect (OR 1.8, CL 1.0-3.3). With respect to end-of-life radiotherapy, because melanoma demonstrated a high event rate (supp. Table 1), it was made a separate level in the regression model (OR 1.9, CL 1.0-3.5). Most cancer types have a similar use of end-of-life radiotherapy.

Table 2 Summary of end-of-life radiotherapy prescriptions

\begin{tabular}{lcc}
\hline \multicolumn{2}{c}{ Number } & Col\% \\
\hline No. of radiotherapy prescriptions per decedent $(N=458)$ & \\
1 & 371 & 81 \\
$2-3$ & 86 & 19 \\
No. of prescriptions $(N=542)$ completed as planned & \\
\multicolumn{2}{l}{$359 / 542$} \\
No. of fractions per prescription $(N=542)$ & 66 \\
Single & 103 & \\
2-5 (mostly 5) & 226 & 19 \\
6-10 (mostly 10) & 116 & 42 \\
$>10$ & 67 & 21 \\
Unclear & 30 & 12 \\
Radiotherapy planning $(N=542)$ & & 6 \\
Simple (e.g., single-field) & 403 & 74 \\
Complex (e.g., 3D) & 92 & 17 \\
Unclear & 47 & 9 \\
Radiotherapy volume/indications $(N=542)$ & & 30 \\
Bone & 210 & 39 \\
Lung/mediastinum & 99 & 18 \\
Brain & 71 & \\
Other & 162 & \\
\hline
\end{tabular}

One prescription per radiotherapy volume of treatment; some patients require multiple volumes of radiotherapy for symptom palliation
Gastrointestinal cancers (ORs 0.10 to 0.54 ) and leukemias (OR 0.20 ) demonstrate very low tendencies for end-of-life radiotherapy, consistent with the infrequent clinical indication for this modality in advanced stages of these diseases.

In both multivariable models, acute care admission is strongly associated with (OR 2.6, CL 2.0-3.4 and OR 6.0, CL 4.7-7.7, respectively) end-of-life cancer therapy. Younger age and decedents with a short duration of disease (16 months) are also more likely to be given cancer therapy at end-of-life. Higher comorbidity scores were associated with less end-of-life radiotherapy. Male decedents are more likely than female to have undergone end-of-life chemotherapy (OR 1.3, CL 1.01-1.8). A trend towards a reduction of end-of-life radiotherapy in the second half of the study (2007-2010) can be appreciated (OR 0.86, CL 0.71-1.05), but no time period effect is apparent for end-of-life chemotherapy.

The effects of cancer type and covariates on hospital death are presented in Fig. 3. Most cancer types show similar tendencies towards hospital death, the exception being a strong tendency among hematological cancer decedents to die in hospital (leukemias OR 3.0, CL 2.2-4.0; lymphomas-myelomas OR 2.2, CL 1.7-2.8). Notably, exposure to either chemotherapy or radiotherapy at end-of-life occurred in $7.4 \%$ (732/9863) of the decedents; this factor is the strongest predictor for dying in hospital (OR 5.1, CL 4.3-6.0). Seventy-seven percent (566/732) of the end-of-life cancer therapy occurred during hospital admission or within close temporal proximity of each other (both events occurring within 2 weeks of death); rarely did the decedents receive both chemotherapy and radiotherapy $(0.2 \%, 22 /$ 9863). Older patients were less likely to die in hospital (OR 0.83 , CL $0.80-85$, per decade increase). Higher comorbidity scores increased the probability of hospital death. A modest time trend was observed, with a lower tendency towards hospital deaths in the second half of the study period (OR 0.80, CL $0.73-0.88$ ). 


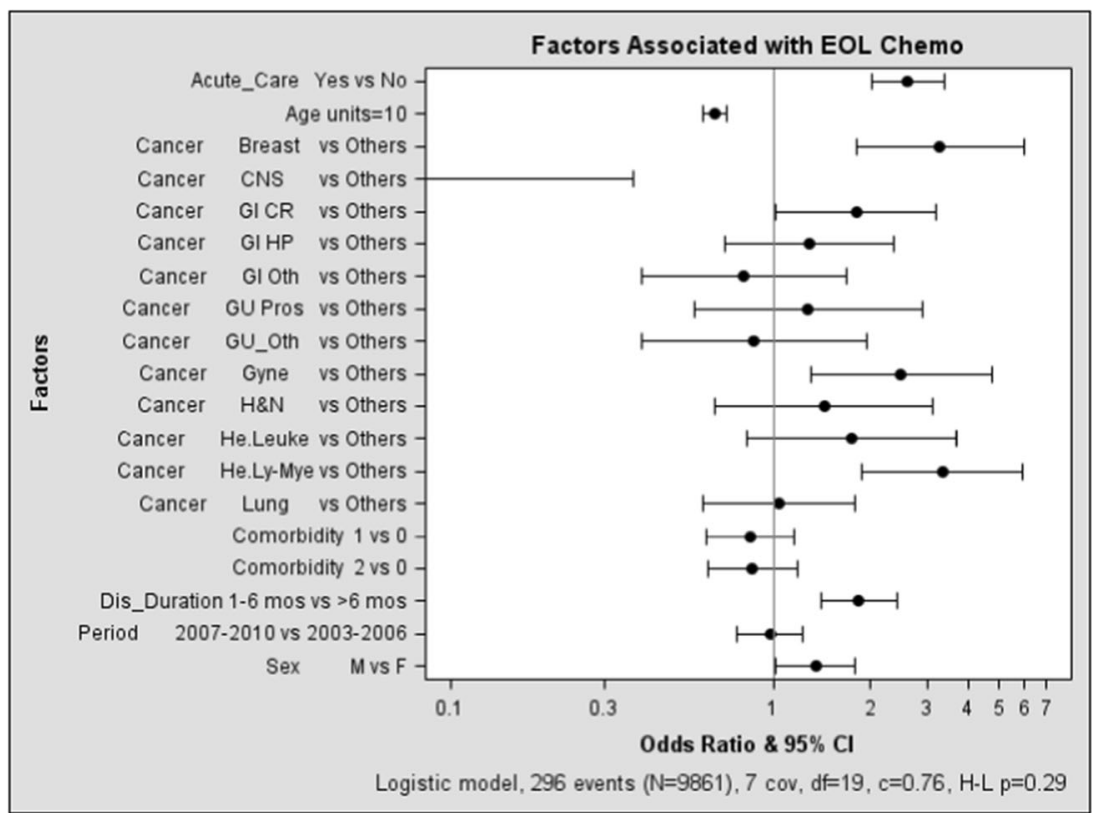

Fig. 1 Multivariable logistic regression of factors associated with end-oflife chemotherapy. Age as continuous variable, OR 0.66 per decade increase. Comorbidity score missing for two cases; Comorbidity "2" covers scores 2 to 8 . Cancer CNS has zero EOL chemo event $(\mathrm{OR}=$ $0.05,95 \% \mathrm{Cl}<0.001$ to 0.37 ). Cancer $G I C R$ colorectal and anus; $G I$ $H P$ hepatobiliary and pancreas; GI Oth upper GI and other GI cancers; Gyne ovarian, cervical, uterine, and other female genital organs; $H \& N$

\section{Summary of findings and interpretation}

In this health services evaluation of end-of-life cancer care at an urban Canadian tertiary referral center, we found that while end-of-life chemotherapy and end-of-life radiotherapy rates were low (3.0 and $4.6 \%$, respectively), the rate of new chemotherapy use in the final month of life (2.2\%) and the rate of head and neck; He.Leuke hematology leukemias; He.Ly-Myel hematology lymphomas, myelomas, and immune disorders; Dis Duration disease duration from date of initial cancer diagnosis to date of death; Acute_Care admission to acute care facility within 14 days of death; $H-L$ Hosmer-Lemeshow goodness-of-fit test; cov covariates; $d f$ degrees of freedom

hospital death (34\%) suggest that end-of-life care was still aggressive. The rate of end-of-life chemotherapy for deaths between 2003 and 2010 in our study is comparable to that reported for Ontario (2.9\% end-of-life chemotherapy by 2004) [8], but variations in the utilization of cancer therapy were considerable across different cancer types and the variations were independent of other relevant variables. End-of-life cancer therapy is itself a
Fig. 2 Multivariable logistic regression of factors associated with end-of-life radiotherapy. Model includes additional level of Cancer: Melan melanoma. Age as continuous variable, odds ratio 0.82 (95\% CI 0.76 to 0.88$)$ per decade increase. See Fig. 1 for abbreviations

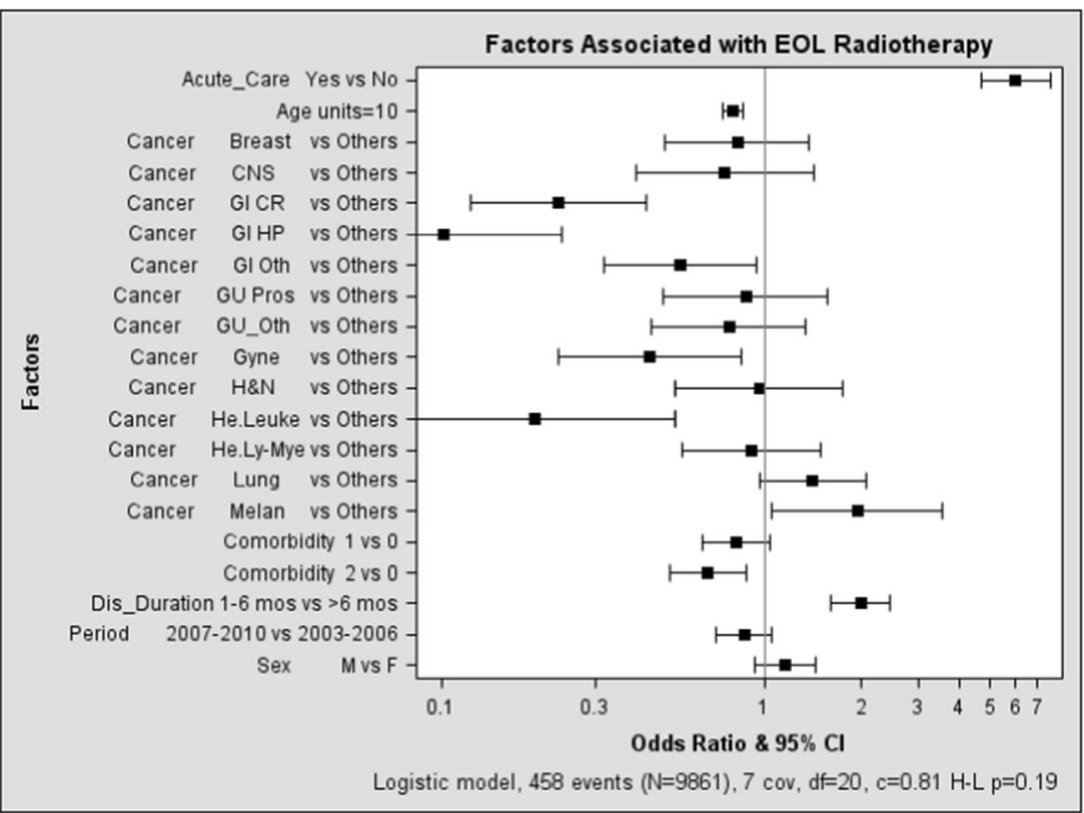


Fig. 3 Multivariable logistic regression of factors associated with dying in hospital. Age as continuous variable, OR 0.83 (95\% CI 0.80 to 0.85$)$ per decade increase. See Fig. 1 for abbreviations. EOL Tx end-oflife chemotherapy and/or radiotherapy

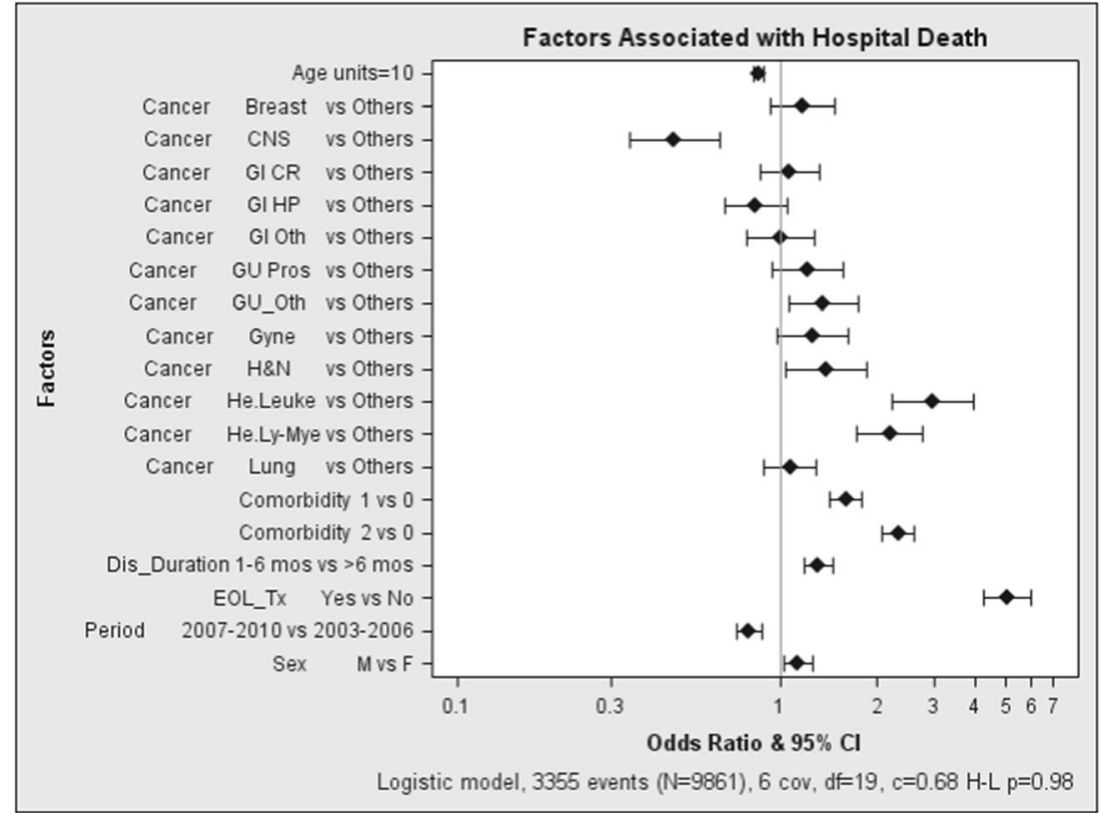

strong predictor of hospital death. Even though the rate of dying in acute care facility appears lower than the national average of $45 \%$ reported for 2011-2012 [15], the $34 \%$ rate of hospital-based death is far from the expressed preference by Albertans to spend end-of-life care at home ( $71 \%$ preferred to be at home near death; $7 \%$ preferred hospital) [16]. The observation that $44 \%$ of decedents requiring acute care hospitalization within 2 weeks of death is concerning for unmet needs among palliative cancer outpatients.

We observed a two- to threefold increase in end-of-life chemotherapy use among breast, hematologic, and gynecological cancers compared to other tumor groups. As Wennberg et al. propose [17], if these variations reflect genuine differences in the informed care preferences of the patients, they may be considered warranted. But based on Wilson et al. [16], one would surmise there are gaps between treatment intensity and end-of-life preferences in our practice, gaps that are not explained by age, comorbidity, and apparent acuity as demonstrated in the multivariable models. The observed variations in end-of-life cancer therapy in this regional cancer program may be unwarranted, and there are strong ethical [18] and economic [19] motivations for closer examinations of practice patterns and decision making processes. In addition, such variations are a source of concern for future cancer management, if they are driven by supply-sensitive care. As new discovery research and drug developments continue, more systemic agents will become available as usual care to the various multidisciplinary tumor groups (most recently, tyrosine kinase inhibitors of various molecular targets). A likely consequence would be an increase in cancer therapy use (and not limited to chemotherapy) in the palliative and end-of-life setting; that is, a normalization of aggressiveness in end-of-life cancer care. In some respects, the notable overprescription of radiotherapy fractions in our study
(34 \% of prescriptions failed to finish as intended) may be a reflection of that normalization. Such overprescription of radiotherapy has been observed elsewhere $[6,7]$ and raises further questions about the supply-sensitive nature of clinical decision making and prescription choices near end-of-life.

We also observed a high acute care admission rate (44\%) before death and a strong association with end-of-life cancer therapy during admission. While further studies are needed to evaluate the nature of the acuity or distress leading to acute care admissions, judicious use of survival prediction tools and prognostic factors, such as those recommended by the European Association of Palliative Care [20], may help identify patients in the final weeks of life for more thorough evaluation of palliative care needs. Decline in performance status over repeated clinic visits [21] may serve as a trigger for reevaluation of goals of therapy and transition to end-of-life care among palliative outpatients. Engagement with patient and family in advance care planning is vital to facilitate their understanding of prognosis, to review their end-of-life preferences, and to manage their expectations, including issues of continuity, treatment discontinuation, and closure [22-24].

A modest time trend was observed towards fewer hospital deaths after 2007 in our study. The time period corresponds to new hospice facilities and initiatives in goals of care designation in acute care. With accumulating evidence towards integrating palliative care services in the ambulatory oncology setting $[25,26]$, future cancer services should see tangible benefits from coordinated efforts to integrate palliative care principles within multidisciplinary tumor group structures. Our group has reported a reduction in end-of-life radiotherapy use after an integrated palliative oncology clinic became available for patients with newly diagnosed brain metastases [27]. In addition to reviewing prognosis, treatment goals, and 
expectations of functional decline, acknowledging psychosocial and existential distress is an essential component of care through this integrated palliative oncology clinic.

\section{Study limitations}

Our study was conducted on decedents managed in an urban, tertiary cancer care setting, and the results are not necessarily representative of rural populations. Clinical factors such as disease extent, prior therapies, symptom distress, and goals of care designation were not determined, and patient heterogeneity has not been described by such factors. As such, the clinical indications and appropriateness of cancer therapy or acute care admission could not be ascertained. The observed chemotherapy use in acute care facilities was low $(0.6 \%)$ but may be underreported by the hospital data management system before electronic drug ordering was implemented in 2007. End-of-life chemotherapy delivery rate, upon verification, was $35 \%$ lower than that identified through administrative data query. To extend our study province-wide, efforts to integrate data from hospital pharmacy, cancer clinic pharmacy, and other parts of cancer services will be essential. Other indicators of aggressive end-of-life care, such as repeated emergency room visits, admission to ICU, and repeated hospital admissions in the final month of life, are being examined. Those results, including the potential impact of prior palliative care consultations and community palliative home care exposure before the final month of life, will be reported separately.

\section{Conclusions}

Cancer patients interact with a highly specialized and intervention-oriented health care system. End-of-life care is often medicalized and intense, and variations in that intensity among different types of cancer may be unwarranted. Multidisciplinary cancer teams are positioned to guide a complex and evolving cancer care program, but enhancement of palliative care training and integration of palliative services are needed to help maintain the balance between interventional and comfort measures in patients through the final phases of their cancer journey.

Acknowledgments The authors thank the following individuals for their advice and contributions to this study: Tom Snodgrass for consultative input on Alberta Cancer Registry data reporting and queries, Margaret Ngo and Carole Chambers for TBCC pharmacy chemotherapy delivery verification, Kevin Lonergan and Cathy Askin for data queries through acute care data management (SCM/DIMR), and Dr. Trafford Crump and Dr. Marc Kerba for insightful comments on the manuscript.

Conflict of interest Drs. Grendarova, Sinnarajah, Trotter, and Wu have no conflict of interest to declare. Dr. Card has provided consultant/ advisory services to Pfizer and Bayer companies unrelated to this study.
Funding Dr. Grendarova is enrolled in the Clinical Investigator Program, University of Calgary, funded by the Alberta Cancer Foundation Clinical Fellowship Dr. Anthony Fields Award.

Open Access This article is distributed under the terms of the Creative Commons Attribution Noncommercial License which permits any noncommercial use, distribution, and reproduction in any medium, provided the original author(s) and the source are credited.

\section{References}

1. The quality of death: ranking end-of-life care across the world. A report from the Economist Intelligence Unit commissioned by Lien Foundation (2010). Available: http://www.eiu.com/site_info.asp? info name $=$ qualityofdeath lienfoundation $\&$ page $=$ noads $\& \mathrm{rf}=0$

2. Earle CC, Neville BA, Landrum MB et al (2005) Evaluating claimsbased indicators of the intensity of end-of-life cancer care. Int J Qual Health Care 17(6):505-509

3. Earle CC, Landrum MB, Souza JM et al (2008) Aggressiveness of cancer care near the end of life: is it a quality-of-care issue? J Clin Oncol 26(23):3860-3866

4. Morden NE, Chang CH, Jacobson JO et al (2012) End-of-life care for Medicare beneficiaries with cancer is highly intensive overall and varies widely. Health Aff 31(4):786-796

5. Guadagnolo BA, Liao KP, Elting L et al (2013) Use of radiation therapy in the last 30 days of life among a large population-based cohort of elderly patients in the United States. J Clin Oncol 31(1):80 87

6. Kapadia NS, Mamet R, Zornosa C et al (2012) Radiation therapy at the end of life in patients with incurable nonsmall cell lung cancer. Cancer 118(17):4339-4345

7. Toole M, Lutz S, Johnstone PA (2012) Radiation oncology quality: aggressiveness of cancer care near the end of life. J Am Coll Radiol 9(3):199-202

8. Ho TH, Barbera L, Saskin R et al (2011) Trends in the aggressiveness of end-of-life cancer care in the universal health care system of Ontario, Canada. J Clin Oncol 29(12):1587-1591

9. Alberta Health Services cancer guidelines, information for health professionals: Available: http://www.albertahealthservices.ca/ cancerguidelines.asp. Accessed Aug 2014

10. Danielson B, Winget M, Gao Z et al (2008) Palliative radiotherapy for women with breast cancer. Clin Oncol (R Coll Radiol) 20(7):506512

11. North American Association of Central Cancer Registries: Available: http://www.naaccr.org/Certification/WhoisCertified.aspx. Accessed Aug 2014

12. Barbera L, Paszat L, Chartier C (2006) Indicators of poor quality endof-life cancer care in Ontario. J Palliat Care 22(1):12-17

13. Deyo RA, Cherkin DC, Ciol MA (1992) Adapting a clinical comorbidity index for use with ICD-9-CM administrative databases. J Clin Epidemiol 45:613-619

14. Quan H, Sundararajan V, Halfon $P$ et al (2005) Coding algorithms for defining comorbidities in ICD-9-CM and ICD-10 administrative data. Med Care 43:1130-1139

15. Canadian Institute for Health Information. End-of-life hospital care for cancer patients. 2013; Available at: https://secure.cihi.ca/free products/Cancer Report EN web April2013.pdf. Accessed 1 May 2013

16. Wilson DM, Cohen J, Deliens L et al (2013) The preferred place of last days: results of a representative population-based public survey. J Palliat Med 16(5):502-508 
17. Wennberg JE, Fisher ES, Skinner JS (2002) Geography and the debate over medicare reform. Health Aff. doi:10.1377/hlthaff.w2.96

18. Moulton B, King JS (2010) Aligning ethics with medical decisionmaking: the quest for informed patient choice. J Law Med Ethics 38(1):85-97

19. Lee EO, Emanuel EJ (2013) Shared decision making to improve care and reduce costs. N Engl J Med 368(1):6-8

20. Maltoni M, Caraceni A, Brunelli C et al (2005) Prognostic factors in advanced cancer patients: evidence-based clinical recommendations - a study by the Steering Committee of the European Association for Palliative Care. J Clin Oncol 23(25):6240-6248

21. Sutradhar R, Barbera L (2014) A Markov multistate analysis of the relationship between performance status and death among an ambulatory population of cancer patients. Palliat Med 28(2):184-190

22. Wright AA, Zhang B, Ray A et al (2008) Associations between endof-life discussions, patient mental health, medical care near death, and caregiver bereavement adjustment. JAMA 300(14):1665-1673
23. Back AL, Young JP, McCown E et al (2009) Abandonment at the end of life from patient, caregiver, nurse and physician perspectives. Arch Intern Med 169(5):474-479

24. Heyland DK, Barwich D, Pichora D et al (2013) Failure to engage hospitalized elderly patients and their families in advance care planning. JAMA Intern Med 173:778-787

25. Temel JS, Greer JA, Muzikansky A et al (2010) Early palliative care for patients with metastatic non-small-cell lung cancer. N Engl J Med $363: 733-742$

26. Smith TJ, Temin S, Alesi ER et al (2012) American Society of Clinical Oncology provisional clinical opinion: the integration of palliative care into standard oncology care. J Clin Oncol 30(8): $880-887$

27. Jung H, Sinnarajah A, Enns B et al (2013) Managing brain metastases patients with and without radiotherapy: initial lessons from a teambased consult service through multidisciplinary integrated palliative oncology clinic. Support Care Cancer 21:3379-3386 